\title{
Segurança da risperidona em crianças e adolescentes com transtorno do espectro autista
}

\author{
Safety of risperidone in children and adolescents with autistic \\ spectrum disorder
}

Recebido em: $16 / 10 / / 2020$ Aceito em: $\quad 09 / 03 / 2021$
Kelly Rose Tavares NEVES; Isabelle Magalhães ALBUQUERQUE; Emanuel Lucas Pinheiro XAVIER; Sophia de Oliveira MARTINS; Gislei Frota ARAGÃO Universidade Federal do Ceará, Núcleo de Pesquisa e Desenvolvimento de Medicamentos-NPDM. Rua Coronel Nunes de Melo, 1000, Rodolfo Teófilo, CEP 60.430-275. Fortaleza, CE, Brasil. E-mail:kelly.rose@hotmail.com

\section{ABSTRACT}

Autism spectrum disorder (ASD) is a neurological development disorder with social, communicative, and behavioral impairments. Risperidone is an antipsychotic medication that has been used for symptom relief and behavioral improvement of ASD. However, several adverse effects have been identified. This work aimed to conduct a literature review regarding the adverse effects of risperidone, specifically in individuals with ASD. Comprehensive searches were performed in the MedLine, PsycInfo, and Science Direct databases, using the descriptors: "Autism Spectrum Disorder", "Risperidone," and "Drug Related Side Effects and Adverse Reactions". Through this search, 29 articles were selected, published between 2000 and 2019. The results showed heterogeneity of adverse reactions, the most prevalent of which were: increased appetite and weight gain, drowsiness, hyperprolactinemia, gastrointestinal symptoms, tachycardia, and extrapyramidal effects. Most are mild or moderate and well. However, more recent studies indicate that there are characteristics of autism that can influence the response to risperidone and increase the risk of adverse reactions, mainly linked to genetic polymorphisms, to the sharing of concomitant medical conditions drug interactions, among others. Thus, considering the complexity of ASD and the vulnerability of autistic children and young people, risperidone should be used with caution in this population. Preliminary screening, monitoring of risk factors, and monitoring of use should be done, especially when prolonged use is necessary.

Keywords: autistic spectrum disorder; risperidone; drug-related side effects and adverse reactions.

\section{RESUMO}

O transtorno do espectro autista (TEA) é um distúrbio de desenvolvimento neurológico, apresentando comprometimentos de ordem sócio, comunicativa e comportamental. A risperidona é um medicamento antipsicótico que tem sido usado para alívio dos sintomas e melhoria comportamental do TEA, no entanto, vários efeitos adversos foram identificados. O objetivo deste trabalho foi realizar uma revisão da literatura sobre os efeitos adversos da risperidona especificamente em indivíduos com TEA. Foram realizadas buscas 
abrangentes nas bases de dados MedLine, PsycInfo e Science Direct, utilizando os descritores: "Autism Spectrum Disorder", "Risperidone" e "Drug Related Side Effects and Adverse Reactions". Por meio desta busca foram selecionados 29 artigos, publicados entre 2000 e 2019. Os resultados mostraram uma heterogeneidade de reações adversas, sendo as mais prevalentes: aumento de apetite e ganho de peso, sonolência, hiperprolactinemia, sintomas gastrointestinais, taquicardia e efeitos extrapiramidais. A maioria delas apresenta grau leve ou moderado e bem gerenciável. Entretanto, estudos mais recentes indicam que existem características do autismo que podem influenciar a resposta à risperidona e elevar o risco de surgimento de reações adversas, principalmente ligadas aos polimorfismos genéticos, ao compartilhamento de condições médicas concomitantes, às interações medicamentosas, entre outros. Dessa forma, considerando a complexidade do TEA e a vulnerabilidade de crianças e jovens autistas, esse medicamento deve ser usado com cautela nessa população, com prévia triagem, acompanhamento dos fatores de risco e monitoramento do uso, em especial quando for necessário uso prolongado.

Palavras-chave: transtorno do espectro autista; risperidona; efeitos colaterais e reações adversas relacionados a medicamentos.

\section{INTRODUÇÃO}

O uso de medicamentos psicotrópicos, especialmente a risperidona, é frequente entre crianças e adolescentes com Transtorno do Espectro Autista (TEA) (1). O TEA é um distúrbio do desenvolvimento neurológico, caracterizado por déficits na comunicação social (socialização e comunicação verbal e não verbal) e no comportamento (interesses restritos, movimentos repetitivos) (2). As causas do autismo são majoritariamente genéticas (3) e até o momento não existem terapias curativas disponíveis. As abordagens de tratamento geralmente incluem intervenções comportamentais que proporcionam uma melhor adaptação ao meio em que o paciente vive, ajudando na melhoria da qualidade de vida do indivíduo (4). A terapia medicamentosa pode ser usada, no entanto, para tratar comportamentos perturbadores geralmente associados ao autismo (ansiedade, irritabilidade, sintomas obsessivo-compulsivos, distúrbios do sono, agressão e autolesão), que interferem negativamente no sucesso dos tratamentos educacionais e qualidade de vida familiar $(5,6)$.

Nesse sentido, a risperidona é o antipisicótico mais popularmente prescrito para crianças com TEA (1), sendo considerada eficaz e bem tolerada principalmente no tratamento de agressividade, hiperatividade, irritabilidade e comportamentos autolesivos (7). Ela faz parte do grupo de antipsi- cóticos conhecidos como atípicos ou de segunda geração, que agem como antagonista dos receptores da dopamina e serotonina, e são reconhecidos pelo menor risco de incidência de efeitos extrapiramidais comparados aos antipsicóticos de primeira geração (8).

Apesar de segura, a risperidona pode provocar efeitos colaterais importantes (9). Alguns são leves, outros, contudo, podem ser intensos e desagradáveis (10). E isto é especialmente preocupante na população com TEA, pois pode experimentar um número maior de reações adversas mesmo em pequenas doses de medicamentos (11). Crianças e adolescentes com TEA também podem apresentar sensibilidade e intensidade aumentada a reações adversas a medicamentos, mesmo em doses mínimas, em comparação com crianças sem TEA (12).

Nesse sentido, o objetivo deste estudo foi apresentar uma revisão da literatura sobre as principais reações adversas à risperidona em crianças e adolescentes com TEA e descrever os possíveis fatores que podem contribuir para a vulnerabilidade dessa população frete às reações.

\section{MÉTODO}

Este artigo é uma revisão narrativa, operacionalizada mediante a busca eletrônica de artigos científicos publicados entre os anos de 2000 e 2019 nas bases de dados MedLine, PsycInfo e Science 
Direct. Os descritores usados para a busca, de forma individual e combinada, foram: "Autism Spectrum Disorder", "Risperidone" e "Drug Related Side Effects and Adverse Reactions".

A amostra compreendeu publicações selecionadas da leitura prévia dos títulos e resumos que versavam sobre os efeitos adversos, efeitos colaterais e toxicidade da risperidona e tendo como participantes indivíduos com TEA, publicados dentro do período estabelecido, escritos na língua inglesa. Inicialmente foram encontrados 128 artigos potenciais para análise, dos quais 12 extraídos da base de dados PubMED, 72 da Science Direct e 44 da
PsycINFO. Foram excluídos estudos publicados sob a forma de editoriais, casos clínicos e estudos pré-clínicos. Posteriormente foi realizada a leitura dos textos na integra, com exclusão daqueles que não tinha pertinência com o tema, ou que não apresentavam o desfecho requerido (reações adversas), ou devido à heterogeneidade da população estudada (compostos por grupos combinados, que incluíam outros transtornos mentais, como esquizofrenia e transtorno-obsessivo-compulsivo). A Figura 1 mostra o processo de seleção dos artigos em suas diferentes etapas e o respectivo número de artigos excluídos em cada uma.

Figura 1. Estratégia de busca e seleção dos artigos para a revisão.

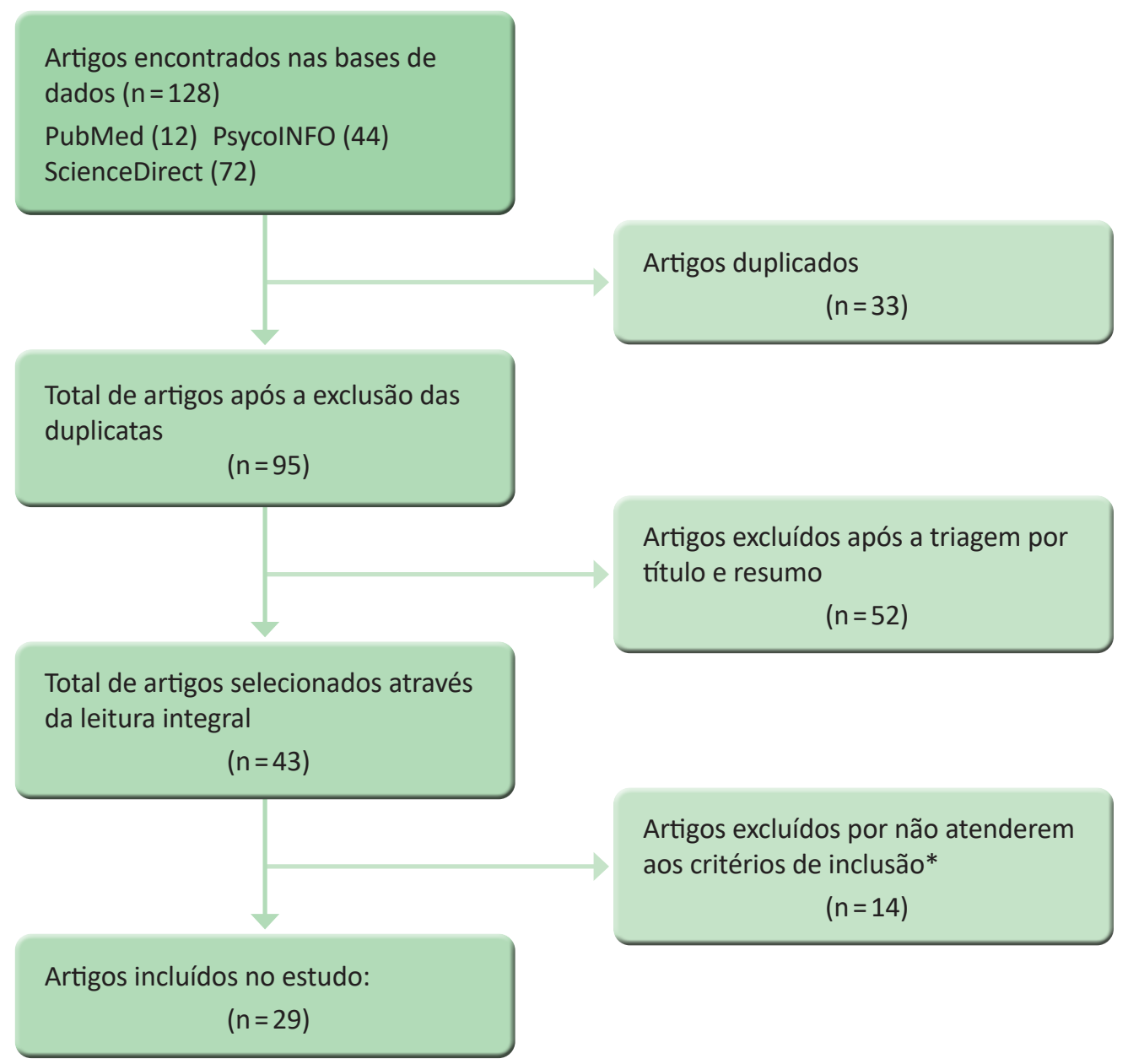

\footnotetext{
*Foram excluídos pelos seguintes motivos: a) aqueles artigos que não tinha pertinência com o tema; b) que não apresentavam o desfecho requerido (reações adversas), ou c) devido à heterogeneidade da população estudada (compostos por grupos combinados, que incluíam outros transtornos mentais, como esquizofrenia e transtorno-obsessivo-compulsivo).
} 
Desse modo, foram selecionados e analisados 29 estudos, os quais foram utilizados na produção desta revisão. No entanto, também se buscou incluir pesquisas que não tratavam o tema desta revisão como central, abordandoos para fundamentar a discussão dos resultados.

\section{RESULTADOS E DISCUSSÃO}

A população estudada nos artigos foi composta de crianças e adolescentes com TEA, com faixa etária mais frequente foi 5 a 17 anos. Os participantes do sexo masculino foram maioria em todos os estudos, o que é justificado pela diferença entre os gêneros no TEA, que é de cerca de 4 vezes mais meninos que meninas, de acordo com dados do Rede de Monitoramento do Autismo e Deficiências do Desenvolvimento (13).

A incidência das reações adversas nos artigos que continham esta informação variou de 49 a $100 \%$ (14-16) entre os pacientes tratados com risperidona. $\mathrm{O}$ número de participantes que se retiraram antes da conclusão dos estudos foi fornecido por quatro artigos, e os motivos foram: ganho de peso excessivo (17), falta de eficácia (18), choro e agitação graves após tomar risperidona (19) e resposta insuficiente (20).

Há grande heterogeneidade de reações adversas à risperidona, sendo as principais: aumento do apetite e ganho de peso, sonolência, hiperprolactinemia, sintomas gastrintestinais, taquicardia e efeitos extrapiramidais.

Aumento do apetite e ganho de peso. $\mathrm{O}$ aumento do apetite e o ganho de peso são reações adversas frequentes à risperidona e parece ter relação com a duração do tratamento, considerando que estudos de curta duração, como os ensaios clínicos controlados, podem não identificar mudanças significativas no peso dos indivíduos tratados com risperidona em comparação com os que usaram placebo (16).

Quando comparado com aripiprazol, um antipsicótico também usado no tratamento de autismo que age como agonista parcial dos receptores D2 de dopamina e 5-HT1A de serotonina e como antagonista do receptor 5-HT2A, o ganho de peso é semelhante entre as crianças e adolescentes com TEA dos dois grupos, ao fim de dois meses de tratamento (19).
Em média, o ganho de peso fica em torno de $2,5 \mathrm{~kg}$ após oito semanas de tratamento com risperidona, 4,2 $\mathrm{kg}$ após 16 semanas, e 5,4 $\mathrm{kg}$ após 24 semanas (21), mas podem atingir 6,2 $\mathrm{kg}$ após 16 semanas (17). Outro estudo mostrou um ganho de peso médio de $8,2 \mathrm{~kg}$ entre crianças de 8 a 12 anos, $8,4 \mathrm{~kg}$ entre adolescentes e 5,4 kg entre adultos, todos acompanhados durante um ano (22).

Além disso, a circunferência da cintura aumenta em média $6 \mathrm{~cm}$, sugerindo aumento no tecido adiposo visceral (21), que pode gerar uma serie de consequências, como o surgimento da síndrome metabólica, diabetes tipo 2, triglicerídeos elevados e hipertensão, que, consequentemente, aumentam o risco de doenças hepáticas e cardiovasculares (27).

As crianças com TEA têm maior risco de desenvolver obesidade [ou seja, índice de massa corporal (IMC) para o percentil 95 da idade] ou sobrepeso (ou seja, IMC para o percentil 85 da idade) do que crianças com desenvolvimento típico (23). Esses valores de IMC estão associados a resultados adversos à saúde, incluindo resistência à insulina, diabetes, doenças cardíacas e certos tipos de câncer (24). A prevalência de obesidade entre crianças com TEA fica em torno de $22,2 \%$ e $37 \%$, com um risco $41,1 \%$ maior de desenvolver obesidade em comparação com crianças sem TEA (25).

Alguns fatores-chave, que podem desempenhar um papel mediador nas taxas mais altas de obesidade, observados em crianças com TEA e incluem comportamentos alimentares (dietas seletivas), estilo de vida sedentário, condições médicas concomitantes e uso de medicamentos, como a risperidona, assim como, a diversidade reduzida da microbiota intestinal, os desequilíbrios hormonais e distúrbios metabólicos (26). Fatores ambientais como a disponibilidade e acesso facilitado aos alimentos e a falta de exercícios físicos (devido a questões como: dificuldades sociais, déficits motores e maior necessidade de supervisão) também podem contribuir para o ganho de peso não saudáveis em crianças com TEA(22).

Portanto, é uma reação adversa preocupante, sobretudo com exposição prolongada, pela possibilidade de afetar funcionamento físico, emocional e social de indivíduos com TEA, prejudicando suas atividades acadêmicas e terapêuticas, agravando in- 
capacidades e reduzindo a qualidade de vida, tanto do indivíduo quanto dos cuidadores (28). Além do mais, pesquisadores chamam atenção para a falta de conhecimento e a necessidade de estudos adicionais para avaliar o grau e a persistência do ganho de peso com o uso de medicamentos em crianças com TEA, principalmente em casos de uso prolongado (26).

Sonolência. A sonolência é um dos efeitos adversos mais frequentes com o uso da risperidona e também o que mais provoca descontinuação do tratamento. Ela está fortemente associada ao insucesso no tratamento com risperidona $(9,30)$ e trata-se de um efeito adverso indesejado e preocupante pois está relacionado ao aumento do risco de acidentes, além contribuir para a diminuição do aproveitamento das atividades terapeuticas, escolares e esportivas. Sua ocorrência entre crianças e adolescentes com TEA que usam risperidona varia de $20,7 \%$ a $74 \%$ versus $7 \%$ a $7,7 \%$ dos que usaram apenas placebo $(16,18-20)$. A risperidona provoca mais sonolência que outros antipsicóticos, como a memantine (33\% vs 13\%) (29).

É possível que a sonolência provocada pela risperidona seja um efeito mais comum no início do tratamento, pois pacientes que nunca tomaram este medicamento apresentam mais sonolência quando entram em contato pela primeira vez do que aqueles que já tinha feito uso (9). A risperidona induz um aumento médio de 40 minutos de sono diurno nas quatro primeiras semanas de tratamento, diminuindo a partir das sexta e oitava semanas (18).

Além disso, crianças e adultos com sintomas autistas geralmente apresentam distúrbios do sono e alterações no ritmo circadiano (31), com taxas que variam de 50 a $80 \%$ dos indivíduos com TEA em comparação com 9 a 50\% no grupo sem TEA (32). Anormalidades genéticas no sistema circadiano, assim como na expressão de genes que regulam as vias da melatonina resultando em baixos níveis de melatonina, podem ser responsáveis por distúrbios circadianos do sono em indivíduos com TEA (33).

O tratamento com melatonina parece melhorar os distúrbios do sono na maioria de crianças e adultos com TEA (34). Os efeitos adversos da melatonina incluem excesso de sonolência diurna, dor de cabeça, tontura e outros distúrbios relacionados (35). Considerando que muitos indivíduos com TEA podem fazer uso concomitante de risperidona e de melatonina, não se pode descartar a possibilidade de interação entre essas drogas $(31,36)$, com exacerbação dos efeitos adversos, em especial a sonolência diurna.

Hiperprolactinemia. Nos estudos incluídos nesta revisão, a prevalência de hiperprolactinemia em crianças e adolescentes com TEA em uso de risperidona é considerado alta e gira em torno de 40 a $50 \%$ dos participantes (37-39) e a magnitude das elevações dos níveis de prolactina em diversos estudos varia de duas a quatro vezes, e este efeito na prolactina provavelmente está relacionado à dose (4).

Comparado com o haloperidol, o aumento da prolactina é maior no grupo de crianças e adolescentes ( 8 a 18 anos) com autismo tratadas com a risperidona, após período de 12 semanas (40).

No fígado, a risperidona é metabolizada pelo citocromo P450 2D6 (CIP2D6) no seu principal metabólito ativo, a 9-hidroxirisperidona, e a concentração deste metabólito é significativamente maior em indivíduos com TEA que desenvolvem hiperprolactinemia, confirmando a correlação entre os níveis de prolactina e a concentração plasmática de risperidona (41).

Um estudo revelou que os níveis séricos de prolactina no grupo crianças e adolescentes com TEA que usavam doses altas da risperidona foi significativamente maior do que nos grupos que usavam doses recomendadas e baixas de risperidona e verificou que quanto mais alta a dose de risperidona, maior é o aumento no nível médio de prolactina sérica. Entretanto, mesmo com o uso de doses recomendadas pelo Food and Drug Administration (FDA) para pacientes pediátricos, o tratamento com risperidona pode causar hiperprolactinemia (39).

Os efeitos a curto e a longo prazo do tratamento com risperidona na prolactina sérica foram avaliados em crianças e adolescentes com autismo. Após 8 semanas de tratamento, a prolactina aumentou para 39,0 $\pm 19,2 \mathrm{ng} / \mathrm{mL}$, em comparação com $10,1 \pm 8,8 \mathrm{ng} / \mathrm{mL}$ para o placebo. Os níveis de prolactina também aumentaram significativamente aos 6 meses $(32,4 \pm 17,8 \mathrm{ng} / \mathrm{mL})$ e aos 22 meses $(25,3 \pm 15,6 \mathrm{ng} / \mathrm{mL})$ de tratamento. Embora os aumentos induzidos pela risperidona tenham a tendência de diminuir com o tempo, os niveis séricos de prolactina ainda permaneceram superior ao nor- 
mal mesmo após 22 meses (38). Outra pesquisa indicou que após 53 meses, metade dos pacientes ainda apresentava hiperprolactinemia (37).

Mesmo assintomática, as consequências a longo prazo de altos níveis de prolactina são sérias (42). Assim, pode causar galactorreia, amenorreia, ginecomastia, hirsutismo, perda de libido, impotência e infertilidade (39) e em tratamentos mais prolongados, a hiperprolactinemia pode levar à diminuição da densidade mineral óssea e osteoporose (38)5-17 years of age.

Uma pesquisa mostrou que a disfunção sexual está presente em $14 \%$ dos indivíduos do grupo de crianças e jovens com TEA (10 a 20 anos) que usavam risperidona, em comparação $0 \%$ dos indivíduos do grupo que não usava, e que meninos tratados com risperidona têm maior probabilidade de relatar diminuição do funcionamento sexual a longo prazo (37). Considerando que os dados estatísticos de autismo continuam mostrando a proporção de quatro meninos para cada menina (13), avaliar a disfunção sexual em adolescentes do sexo masculino usando risperidona de longa duração torna-se imprescindível.

Variações genéticas podem influenciar o surgimento de efeitos adversos metabólicos da risperidona. Por exemplo, o polimorfismo CIP2D6, pode alterar o processo de biotransformação da risperidona, gerando variabilidade na sua concentração plasmática, bem como do 9-hidroxirisperidona, que pode resultar falha terapêtica e toxicidade $(41,43)$. Outros polimorfismos genéticos, incluindo o gene do receptor da dopamina (DRD2), o gene do receptor da serotonina e o gene da glicoproteína $\mathrm{P}$ também podem desempenhar um papel significativo na hiperprolactinemia em crianças e adolescentes com TEA (44). Sabe-se que o TEA tem fortes bases genéticas; assim, a influência de variações genéticas da resposta à risperidona pode ter ligações compartilhadas em alguns indivíduos com TEA e isso precisa ser investigado.

Sintomas gastrintestinais (GI). Os sintomas gastrintestinais (GI) mais comuns foram constipação, náusea, vômito, dispepsia e elevação das enzimas hepáticas, em especial alanina aminotransferase (ALT) e aspartato aminotransferase (AST). A frequência dos sintomas do trato GI em crianças com TEA varia de 9 a 84\%, dependendo do método (estudos retrospectivos e prospectivos), em comparação com $9 \%$ a $37 \%$ em crianças sem TEA (45). Os sintomas gastrointestinais incluem superprodução de gases intestinais / flatulência (60\%), inchaço (38\%), dor abdominal (378\%), diarreia (28\%), arrotos $(25 \%)$, sintomas de refluxo gastroesofágico (16\%) e constipação (10\%) (46).

O diagnóstico de distúrbio gastrointestinal em crianças com TEA pode ser mais difícil e tardio, em comparação com crianças sem TEA, pois, sintomas subjetivos, como dor, desconforto, azia ou náusea, são muito difíceis de avaliar e interpretar devido às dificuldades na comunicação verbal e não verbal, além de uma percepção alterada da dor $(47,48)$.

A associações entre sintomas GI e sintomas de TEA foram analisadas em alguns estudos. Crianças com TEA que apresentaram esofagite de refluxo exibiram irritabilidade inexplicável com mais frequência (43\%) do que aquelas que não apresentaram (13\%) (49). Em um dos estudos, o tipo mais comum de distúrbio gastrointestinal em crianças com TEA $(85,0 \%)$ foi constipação funcional o qual estava significativamente associado ao comprometimento da linguagem (71).

Esses sintomas podem contribuir para alterações no comportamento e na cognição. Em um estudo usando dados do Autism Genetic Resource Exchange (AGRE), no qual apenas famílias com vários membros afetados estão incluídas, os pais relataram significativamente mais problemas gastrointestinais em crianças com TEA do que em irmãos não afetados ( $42 \%$ vs. $12 \%$ ), além disso, este foi o primeiro estudo a relatar que o aumento da gravidade do autismo foi associado a maiores chances de problemas GI (50).

Dessa forma, a administração de medicamentos que podem causar problemas gastrointestinais, como a risperidona, podem provocar dificuldades adicionais em crianças com TEA que já apresentem distúrbios gastrointestinais (26).

Taquicardia. A risperidona provavelmente não apresenta efeitos cardiovasculares clinicamente relevantes em pacientes sem problemas cardíacos, apesar de os antipsicóticos poderem aumentar o intervalo QT (51). O intervalo QT é um parâmetro mensurado pelo eletrocardiograma (ECG) que corresponde ao período que vai desde o início da 
despolarização até o final da repolarização ventricular (52). Apesar disso, nossa hipótese é que a risperidona pode contribuir para aumento de risco cardíaco, indiretamente, através dos seus efeitos metabólitos, principalmente o aumento de peso e a obesidade, que são fatores de risco conhecidos para diversas doenças cardiovasculares.

A risperidona pode provocar um aumento médio de 8,9 batimentos por minuto (bpm) entre os pacientes com TEA, além de queixas de taquicardia leve a moderada, em comparação com uma diminuição média de 0,6 bpm entre os pacientes tratados com placebo e relatados desse evento adverso (20).

Um estudo sobre os efeitos da risperidona na função cardíaca em crianças com TEA por oito semanas, não identificou nenhum evento adverso por condução cardíaca e não houve diferença na alteração média no intervalo QT em comparação ao placebo (53). Assim como não foram detectados eventos clinicamente significativos medidos por ECG (16), contudo, apesar disso, queixas de taquicardia foram relatadas por pacientes com TEA em tratamento com risperidona, mesmo sem alterações no ECG $(18,54)$.

Além disso, existem evidências que um desequilíbrio regulatório do sistema nervoso autônomo (SNA) em populações com TEA que poderia levar a risco cardíaco (55). Este desequilíbrio autonômico, caracterizado por uma hiperatividade do sistema nervoso simpático e hipoatividade do sistema nervoso parassimpático, está associado a várias condições cardíacas, como taquicardia, hipertensão e um risco aumentado de doença cardiovascular (56).

A variabilidade da frequência cardíaca (VFC) é um índice que mede as variações dos batimentos cardíacos, envolvendo os ramos simpático e parassimpático, e representa a medida da regulação autonômica do coração (57). Dessa forma, existem evidências de que redução da VFC está associada ao risco cardíaco (58). Estudos indicam que indivíduos com TEA apresentam menor VFC em repouso, comparadas com indivíduos tipicamente desenvolvidos $(56,59)$, sugerindo menor atividade parassimpática. E esta característica está ligada não apenas ao autismo, mas também outras condições mentais como: depressão, psicose, ansiedade e transtorno de déficit de atenção (56), que são condições comumente comórbidas com TEA, e que podem requerer tratamento farmacológico. $\mathrm{O}$ uso prolongado de alguns destes medicamentos psicotrópicos (estimulantes e antidepressivos) também é considerado um fator de risco importante que influencia na VFC (60).

Portanto, na pessoa com TEA, a relação entre a função cardíaca e o SNA não deve ser negligenciada no momento de prescrever risperidona.

Efeitos extrapiramidais (EEP). Os efeitos extrapiramidais com o uso da risperidona foram considerados pouco frequentes, ficando entre 6 e $8 \%$ na dose de com menos de $2 \mathrm{mg} / \mathrm{dia}(9,14)$. Entretanto, a partir de $6 \mathrm{mg} /$ dia de risperidona, os pacientes começam a apresentar mais reações adversas a medicamentos, principalmente efeitos extrapiramidais (51). Os distúrbios do movimento, quando relatados em indivíduos que usaram risperidona podem ser discinesia, hipercinesia, hipocinesia, contrações musculares involuntárias e tremor (16).

Os antipsicóticos atípicos, como a risperidona, supostamente têm vantagens importantes sobre os antipisicóticos mais antigos, particularmente em relação à menor incidência de efeitos extrapiramidais $(40,61)$. No entanto, estudo mais recentes forneceram evidências em contrário (62).

A menor propensão ao desenvolvimento de EEP com antipsicóticos atípicos tem sido atribuída à relativamente fraca ligação destes aos receptores D2 em comparação com os antipsicóticos convencionais, como o haloperidol. No entanto, a risperidona é igualmente potente no bloqueio dos receptores D2 e 5-HT2, e sua potência no bloqueio de receptores D2 depende da dose utilizada, possuindo, assim, uma capacidade inata de produzir uma gama de efeitos adversos (62).

Outro alvo importante para a risperidona é o receptor de dopamina D3 (DRD3), no sistema dopaminérgico mesolímbico. O DRD3 é expresso em áreas motoras dos núcleos da base, desempenhando importante papel na geração de movimentos. Recentes estudos associaram a ocorrência de EEP induzido pela risperidona com polimosfismos no gene da DRD3 $(63,64)$. Além disso, poliformorfismos localizados no gene do DRD3 foram encontrados em indivíduos com TEA no Reino Unido, Países Baixos e Espanha e estavam significativamente associados aos comportamentos repetitivos e este- 
reotipados (64). Portanto, indivíduos com TEA que apresentem polimorfismos no DRD3 tem maior risco de desenvolver EEP com o uso de risperidona.

O desenvolvimento de EEP não é um fenômeno uniforme e está associado a uma série de fatores de risco que incluem exposição prolongada a antipsicóticos, aumento da idade, sexo feminino, raça não caucasiana, presença distúrbios afetivos, dano cerebral orgânico, história de diabetes mellitus, abuso de álcool e vulnerabilidade genética (65).

Cerca de $60 \%$ a $80 \%$ das pessoas com autismo têm anormalidades motoras, incluindo hipotonia, planejamento motor deficiente, coordenação pobre e caminhar com as pontas dos pés. Outros estudos mostraram alguns distúrbios do movimento, como ataxia, acinesia, discinesia e bradicinesia em indivíduos com TEA, tanto idiopática quanto sindrômica $(66,72)$. E alguns pesquisadores propõem que o TEA poderia ser, pelo menos em parte, um distúrbio de movimento (67), o que aumenta o risco de desenvolvimento de efeitos extrapiramidais nesta população. Nesse sentido, pacientes com TEA que apresentem alterações da marcha e de postura devem ser avaliados quanto à discinesia tardia usando as escalas de classificação validadas (70).

Portanto, os profissionais de saúde devem estar cientes do risco de desenvolver discinesia tardia
(DT), principalmente em condições que requerem tratamento em longo prazo como o autismo (68). Problemas críticos associados à DT incluem dificuldade em engolir, dificuldade de locomoção, envolvimento dos músculos respiratórios e fala tornado ininteligível, além disso, a DT pode ser extremamente desfiguradora, aumenta o estigma (69).

\section{CONCLUSÃO}

A complexidade do autismo torna tudo à sua volta igualmente complexo. O presente estudo de revisão da literatura destacou que, considerando a heterogeneidade da apresentação clínica, algumas características encontradas em indivíduos com TEA podem desempenhar um papel fundamental no risco de surgimento de reações adversas ao uso de risperidona, como polimorfismos genéticos que podem alterar a farmacocinética ou a farmacodinâmica da risperidona, desequilíbrio hormonal, distúrbios metabólicos, hábitos alimentares, estilo de vida, microbiota intestinal, presença de condições médicas comórbidas e uso de concomitante de outros medicamentos. Dessa forma, a risperidona deve ser usada cautelosamente nesta população após prévia triagem e acompanhamento dos fatores de risco mencionados.

\section{REFERÊNCIAS}

1. Mandel D, Morales K, Marcus S, Stahmer A, Doshi J, Polsky D. Psychotropic medication uses among Medicaid-enrolled children with autism spectrum disorders. Pediatrics. 2008;121:441-448. DOI: 10.1542/ peds.2007-0984.

2. APA. American Psychiatris Association. Diagnostic and Statistícal Manual of Mental Disorders, Fifth Edition. Washington; 2013.

3. Bai D, Yip BHK, Windham GC, Sourander A, Francis R, Yoffe R, Glasson E, Mahjani B, Suominen A, Leonard H, Gissler M, Buxbaum JD, Wong K, Schendel D, Kodesh A, Breshnahan M, Levine SZ, Parner ET, Hansen SN, Hultman C, Reichenberg A, Sandin S. Association of Genetic and Environmental Factors with Autism in a 5-Country Cohort. JAMA Psychiatry. 2019;76(10): 1035-1043. DOI: 10.1001 / jamapsychiatry.2019.1411.

4. Teixeira G. Manual do Autismo. 2nd ed. Rio de Janeiro: Best Seller; 2016. 96p. ISBN: 9788576849674.
5. Weissman LCB. Autism spectrum disorder in children and adolescents: Overview of management. Uptodate. 2013. Disponível em: http://www.uptodate.com

6. SBP. Sociedade Brasileira de Pediatria. Departamento Científico de Pediatria do Desenvolvimento e Comportamento. Manual de Orientação. Transtorno do Espectro do Autismo, v.5, p. 1-24, 2019.

7. Simeon J, Milin R, Walker S. A retrospective chart review of risperidone use in treatment-resistant children and adolescents with psychiatric disorders. Prog Neuropsychopharmacol Biol Psychiatry. 2002;26(2): 267-275. DOI: 10.1016/s0278-5846(01)00264-0.

8. BRASIL, Risperidona no Transtorno do Espectro do Autismo (TEA). Relatório de Recomendação da Comissão Nacional de Incorporação de Tecnologias no SUS - CONITEC - 123. Brasília (DF): Ministério da Saúde. 2014. 
9. Kent JM, Hough D, Singh J, Karcher K, Pandina G. An open-label extension study of the safety and efficacy of risperidone in children and adolescents with autistic disorder. J Child Adolescent Psychopharmacol. 2013;23(10):676-686. DOI: 10.1089/cap.2012.0058.

10. BRASIL. Linha de cuidado para a atenção às pessoas com transtornos do espectro do autismo e suas famílias na Rede de Atenção Psicossocial do Sistema Único de Saúde. Brasília (DF): Ministério da Saúde. 2015.

11. West L., Waldrop J., Brunssen S. Pharmacologic Treatment for the Core Deficits and Associated Symptoms of Autism in Children. Journal of Pediatric Health Care. 2009;23(2):75-89. DOI: 10.1016/j.pedhc.2008.12.001.

12. Sharma A, Shaw S. Efficacy of Risperidone in Managing Maladaptive Behaviors for Children With Autistic Spectrum Disorder: A Meta-Analysis. Journal of Pediatric Health Care. 2012; 26(4):91-299. DOI: 10.1016/j.pedhc.2011.02.008.

13. Baio J, Wiggins L, Christensen DL, Maenner MJ, Daniels J, Warren Z, Kurzius-Spencer M, Zahorodny W, Robinson Rosenberg C, White T, Durkin MS, Imm P, Nikolaou L, Yeargin-Allsopp M, Lee LC, Harrington R, Lopez M, Fitzgerald RT, Hewitt A, Pettygrove S, Constantino JN, Vehorn A, Shenouda J, Hall-Lande J, Van Naarden Braun K, Dowling NF. Prevalence of autism spectrum disorder among children aged 8 years - autism and developmental disabilities monitoring network, 11 sites, United States, 2014. MMWR Surveill Summ 2018;67(SS-6):1-23

14. King B, Zwi K, Nunn K, Longworth J, Dossetor D. Use of risperidone in a paediatric population: An observational study. Journal of Paediatrics and Child Health. 2003; 39(7):523-527. DOI: 10.4088/PCC.18102283.

15. Boon-Yasidhi V, Jearnarongrit P, Tulayapichitchock $\mathrm{P}$, Tarugsa J. Adverse effects of risperidone in children with autism spectrum disorders in a naturalistic clinical setting at siriraj hospital, Thailand. Psychiatry J. 2014; 2014:136158-136162. DOI:10.1155/2014/136158

16. Pandina G, Bossie C, Youssef E, Zhu Y, Dunbar F. Risperidone Improves Behavioral Symptoms in Children with Autism in a Randomized, Double-Blind, PlaceboControlled Trial. J Autism Dev Disord. 2007; 37(2): 367-373. DOI: 10.1007/s10803-006-0234-7.

17. Troost PW., Lahuis BE., Steenhuis MP., Ketelaars CEJ., Buitelaar JK., Van Engeland H., Scahill L, Minderaa RB, Hoekstra PJ. Long-term effects of risperidone in children with autism spectrum disorders: A placebo discontinuation study. J. American Academy of Child and Adolescent Psychiatry. 2005;44(11),1137-1144. DOI: 10.1097/01.chi.0000177055.11229.76.

18. Aman MG, Arnold E, Christopher J, et al. Acute and Long-Term Safety and Tolerability of Risperidone in Children with Autism. J Child Adolesc Psychopharmacol. 2005;15(6):869-884. DOI: 10.1089/cap.2005.15.869

19. Ghanizadeh A., Sahraeizadeh A., Berk M. A head-tohead comparison of aripiprazole and risperidone for safety and treating autistic disorders, a randomized double blind clinical trial. Child Psychiatry and Hum Dev. 2014;45(2):185-192. DOI: 10.1007/s10578-0130390-x.

20. Shea S., Turgay A., Carroll A., Schulz M., Orlik H., Smith I., Dunbar F. Risperidone in the Treatment of Disruptive Behavioral Symptoms in Children With Autistic and Other Pervasive Developmental Disorders. Pediatrics. 2004;114(5): e634-e641. DOI: 10.2147/ndt.s1450.

21. Scahill L, McDougle CJ, Aman MG, Johnson C, Handen B, Bearss K, Dziura J, Butter E, Swiezy NG, Arnold LE, Stigler KA, Sukhodolsky DD, Lecavalier L, Pozdol SL, Nikolov R, Hollway JA, Korzekwa P, Gavaletz A, Kohn AE, Koenig K, Grinnon S, Mulick JA, Yu S, Vitiello B. Research Units on Pediatric Psychopharmacology Autism Network. Effects of risperidone and parent training on adaptive functioning in children with pervasive developmental disorders and serious behavioral problems. J Am Acad Child Adolesc Psychiatry. 2012;51:136-146. DOI: 10.1016/j. jaac.2011.11.010.

22. Hellings J., Zarcone J., Crandall K., Wallace D., Schroeder S. Weight gain in a controlled study of risperidone in children, adolescents and adults with mental retardation and autism. J Child Adolesc Psychopharmacol. 2001; 11(3):229-238. DOI: 10.1089/10445460152595559.

23. Zheng Z, Zhang L, Li S.; Zhao F, Wang Y, Huang L, Huang J, Zou R, Qu Y, Mu D. Association among obesity, overweight and autism spectrum disorder: A systematic review and meta-analysis. Sci. Rep. 2017;7:11697. DOI: 10.1038/s41598-017-12003-4

24. Weihrauch-Blüher S.; Schwarz P.; Klusmann JH.. Childhood obesity: Increased risk for cardiometabolic disease and cancer in adulthood. Metab. Clin. Exp. 2019;92:147-152. DOI: 10.1016/j.metabol.2018.12.001.

25. Kahathuduwa C, West B, Blume J, Dharavath N, Moustaid-Moussa N, Mastergeorge A. The risk of overweight and obesity in children with autism spectrum disorders: A systematic review and meta-analysis. Obes Rev. 2019;20:1667-1679. DOI: 10.1111/obr.12933.

26. Dhaliwal K; Orsso CE; Richard C; Haqq A; Zwaigenbaum, L. Risk Factors for Unhealthy Weight Gain and Obesity among Children with Autism Spectrum Disorder. Int. J. Mol. Sci. 2019,20:3285-3314. DOI: 10.3390/ijms20133285

27. Scahill L, Jeon S, Boorin SJ, McDougle CJ, Aman MG, Dziura J, McCracken JT, Caprio S, Arnold LE, Nicol G, Deng Y, Challa SA, Vitiello B. Weight Gain and Metabolic Consequences of Risperidone in Young Children with Autism Spectrum Disorder. J Am Acad Child Adolesc Psychiatry. 2016;55(5):415-423. doi:10.1016/j.jaac.2016.02.016.

28. Khodaverdi F.; Alhani F.; Kazemnejad, A.; Khodaverdi Z. The Relationship between Obesity and Quality of Life in School Children. Iran. J. Public Health. 2011;40(2): 96-101. 
29. Nikvarz N., Alaghband-Rad J., Tehrani-Doost M., Alimadadi A., Ghaeli P. Comparing efficacy and side effects of memantine vs. risperidone in the treatment of autistic disorder. Pharmacopsychiatry. 2017;50(1): 19-25. DOI: $10.1055 / \mathrm{s}-0042-108449$.

30. Lemmon M, Gregas M, Jeste S. Risperidone use in autism spectrum disorders: A retrospective review of a clinic-referred patient population. J Child Neurol. 2011; 26(4):428-432. DOI: 10.1177/0883073810382143.

31. Carmassi C, Palagini L, Caruso D, Masci I, Nobili L, Vita A, Dell'Osso L. Systematic Review of Sleep Disturbances and Circadian Sleep Desynchronization in Autism Spectrum Disorder: Toward an Integrative Model of a Self-Reinforcing Loop. Front Psychiatry. 2019;10:366-657. DOI: 10.3389/fpsyt.2019.00366

32. Richdale A, Schreck K. Sleep problems in autism spectrum disorders: prevalence, nature, and possible biopsychosocial aetiologies. Sleep Med Rev. 2009; 13(6):403-411. .DOI: 10.1016/j.smrv.2009.02.003

33. Zee PC., Vitiello MV. Circadian rhythm sleep disorder: irregular sleep wake rhythm type. Sleep Med Clin. 2009;4(2):213-218. DOI: 10.1016/j.jsmc.2009.01. 009

34. Galli-Carminati G, Deriaz N, Bertschy G. Melatonin in treatment of chronic sleep disorders in adults with autism: a retrospective study. Swiss Med Wkly. 2009;139(19-20):293-296. DOI: 10.4414/smw.2009. 12342

35. Besag F, Vasey M, Lao K, Wong I. Adverse Events Associated with Melatonin for the Treatment of Primary or Secondary Sleep Disorders: A Systematic Review. CNS Drugs. 2019;312):1167-1186. DOI: 10.1007/ s40263-019-00680-w

36. Andersen I, Kaczmarska J, Mcgrew S, Mallow B. Melatonina para insônia em crianças com distúrbios do espectro do autismo. J Child Neurol. 2008;23(5): 482-485. DOI: $10.1177 / 0883073807309783$

37. Roke Y, Buitelaar J., Boot A., Tenback D, Van Harten P. Risk of hyperprolactinemia and sexual side effects in males 10-20 years old diagnosed with autism spectrum disorders or disruptive behavior disorder and treated with risperidone. J Child Adolesc Psychopharmacol. 2012;22(6):432-439. DOI: 10.1089/cap.2011.0109.

38. Anderson GM, Scahill L, McCracken JT, McDougle CJ, Aman MG, Tierney E, Arnold LE Martin A, Katsovich L, Posey D, Shah B, Vitiello B. Effects of Shortand Long-Term Risperidone Treatment on Prolactin Levels in Children with Autism. Biological Psychiatry. 2007;61(4):545-550.

39. Hongkaew Y., Ngamsamut N., Puangpetch A., Vanwong N., Srisawasdi P., Chamnanphon M., Chamkrachchangpada B, Tan-kam T, Limsila P, Sukasem C. Hyperprolactinemia in Thai children and adolescents with autism spectrum disorder treated with risperidone. Neuropsychiatr Dis Treat. 2015;11:191-196. DOI: 10.2147/NDT.S76276
40. Miral S, Gencer O, Inal-Emiroglu F, Baykara B, Baykara A, DiriK E. Risperidone versus haloperidol in children and adolescents with AD: A randomized, controlled, double-blind trial. Eur Child Adolesc Psychiatry. 2008;17(1):1-8. DOI: 10.1007/s00787-007-0620-5.

41. Ngamsamut N., Homgkaew Y., Vanwong, N., Srisawasdi P., Puangpetch A., Chamkrachangpada B., Tan-Khum T., Limsila P, Sukasem C. 9-Hydroxyrisperidone-Induced Hyperprolactinaemia in Thai Children and Adolescents with Autism Spectrum Disorder. Basic Clin Pharmacol Toxicol. 2016;119(3): 267-272. DOI: 10.1111/bcpt.1257

42. Szarfman A, Tonning JM, Levine JG, Doraiswamy PM. Atypical antipsychotics and pituitary tumors: A pharmacovigilance study. Pharmacotherapy. 2006;26(6): 748-758. DOI: 10.1592/phco.26.6.748.

43. Vanwong N, Ngamsamut N, Hongkaew Y, Nuntamool N, Puangpetch A, Chamnanphon M, Sinrachatanant A, Limsila P, Sukasem C. Detection of CYP2D6 polymorphism using Luminex xTAG technology in autism spectrum disorder: CYP2D6 activity score and its association with risperidone levels. Drug Metab Pharmacokinet. 2016;31(2):156-162. DOI: 10.1016/j. dmpk.2016.01.005.

44. Sukasem C, Hongkaew Y, Ngamsamut N, Puangpetch A, Van-Wong N, Chamnanphon M, Chamkrachchangpada B, Sinrachatanant A, Limsila P. Impact of pharmacogenetic markers of CYP2D6 and DRD2 on prolactin response in risperidone- treated Thai children and adolescents with autism spectrum disorders. J Clin Psychopharmacol. 2016;36:141-146. DOI: 10.1097/ JCP.0000000000000474.

45. Molloy C, Manning-Courtney P. Prevalence of chronic gastrointestinal symptoms in children with autism and autistic spectrum disorders. Autism. 2003;7(2):165-171. DOI: $10.1177 / 1362361303007002004$.

46. Wasilewska J, Klukowski M. Gastrointestinal symptoms and autism spectrum disorder: links and risks - a possible new overlap syndrome. Pediatric Health Med Ther.. 2015;6:153-166. DOI:10.2147/PHMT.S85717

47. Buie T, Campbell DB, Fuchs GJ 3rd, Furuta GT, Levy $\mathrm{J}$, Vandewater J, Whitaker AH, Atkins D, Bauman ML, Beaudet AL, Carr EG, Gershon MD, Hyman SL, Jirapinyo P, Jyonouchi H, Kooros K, Kushak R, Levitt P, Levy SE, Lewis JD, Murray KF, Natowicz MR, Sabra A, Wershil BK, Weston SC, Zeltzer L, Winter H. Evaluation, diagnosis, and treatment of gastrointestinal disorders in individuals with ASDs: a consensus report. Pediatrics. 2010;125(Suppl 1):S1-S18. DOI: 10.1542/ peds.2009-1878C.

48. McElhanon B, McCracken C, Karpen S, Sharp W. Gastrointestinal symptoms in autism spectrum disorder: a meta-analysis. Pediatrics. 2014;133(5):872-883. DOI: 10.1542/peds.2013-3995.

49. Horvath K, Perman J. Autism and gastrointestinal symptoms. Curr Gastroenterol Rep. 2002;4(3):251-258. DOI: $10.1007 / \mathrm{s} 11894-002-0071-6$ 
50. Wang LW, Tancredi DJ, Thomas DW. The prevalence of gastrointestinal problems in children across the United States with autism spectrum disorders from families with multiple affected members. J Dev Behav Pediatr. 2011; 32(5):351-360. DOI: 10.1097/DBP.0b013e31821bd06a.

51. De Leon J, Sandson NB, Cozza KL. A preliminary attempt to personalize risperidone dosing using drugdrug interactions and genetics: Part I. Psychosomatics. 2008;49(3):258-270. DOI: 10.1176/appi.psy.49.4.347.

52. Harrigan E, Miceli J, Anziano R, Watsky E, Reeves K, Cutler N, Sramek J, Shiovitz T, Middle M. A randomized evaluation of the effects of six antipsychotic agents on QTc, in the absence and presence of metabolic inhibition. J Clin Psychopharmacol 2004;24:62-69. DOI: 10.1097/01.jcp.0000104913.75206.62.

53. Vo LC, Snyder C, McCracken C, McDougle CJ, McCracken JT, Aman MG, Tierney E, Arnold LE, Levi D, Kelleman M, Carroll D, Morrissey J, Vitiello B, Scahill L. No Apparent Cardiac Conduction Effects of Acute Treatment with Risperidone in Children with Autism Spectrum Disorder. J Child Adolesc Psychopharmacol. 2016; 26(10):900-908. DOI: 10.1089/cap.2016.0090.

54. Aman MG, et al. Tolerability, safety, and benefits of risperidone in children and adolescents with autism: 21-month follow-up after 8-week placebo-controlled trial. J Child Adolesc Psychopharmacol. 2015;25(6): 482-493. DOI: 10.1089/cap.2015.0005.

55. Porges S. The polyvagal theory: New insights into adaptive reactions of the autonomic nervous system. Cleve Clin J Med. 2009;76(Suppl 2):S86-S90. DOI: 10.3949/ccjm.76. s2.17.

56. Thapa R, Gail A, Zaidi Tooba A, Thomas EE, Hickie IB, Park SH, Guastella AJ. Reduced heart rate variability in adults with autism spectrum disorder. Autism Res. 2019;12(6):922-930. DOI: 10.1002/aur.2104.

57. Alvares G, Quintana D, Kemp A, Zwieten A, Balleine B, Hickie I, Guastella A. Reduced heart rate variability in social anxiety disorder: Associations with gender and symptom severity. PLoS One. 2013;8:7. DOI: 10.1371/ journal.pone.0070468.

58. Hori M., Okamoto, H. Heart rate as a target of treatment of chronic heart failure. J Cardiol. 2012;60(2):86-90. DOI: $10.1016 /$ j.jjcc.2012.06.013.

59. Ellenbroek B., Sengul H. Autism spectrum disorders: Autonomic alterations with a special focus on the heart. Heart and Mind. 2017;1(2):78-83. DOI: 10.4103/ hm.hm_5_17

60. Silke B., Campbell C., King D. The potential cardiotoxicity of antipsychotic drugs as assessed by heart rate variability. J Psychopharmacol. 2002;16:355-360. DOI: $10.1177 / 026988110201600410$.
61. Siegel M, Beaulieu A. Psychotropic medications in children with autism spectrum disorders: a systematic review and synthesis for evidence-based practice. J. Autism Dev. Disord. 2012;42:1592-1605. DOI: 10.1007/ s10803-011-1399-2.

62. Thomson SR, Chogtu B, Bhattacharjee D, Agarwal S. Extrapyramidal Symptoms Probably Related to Risperidone Treatment: A Case Series. Ann Neurosci. 2017;24(3):155-163. DOI: 10.1159/000477153.

63. Gassó P, Mas S, Bernardo M, Alvarez S, Parellada E, Lafuente A. A common variant in DRD3 gene is associated with risperidone-induced extrapyramidal symptoms. Pharmacogenomics J. 2009;9(6):404-410. DOI: $10.1038 /$ tpj.2009.26

64. Staal W. Autism, DRD3 and repetitive and stereotyped behavior, an overview of the current knowledge. Eur Neuropsychopharmacol. 2014;25(9):1421-1426. DOI: 10.1016/j.euroneuro.2014.08.011.

65. Divac N, Prostran M, Jakovcevski I, Cerovac N: Secondgeneration antipsychotics and extrapyramidal adverse effects. Biomed Res Int 2014;2014:656370. DOI: $10.1155 / 2014 / 656370$

66. Bell L, Wittokowski A, Hare D. Movement Disorders and Syndromic Autism: A Systematic Review. J Autism Dev Disord. 2019;49(1):54-67. DOI:10.1007/s10803018-3658-y

67. Nayate A, Bradshaw JL, Rinehart NJ. Autism and Asperger's disorder: are they movement disorders involving the cerebellum and/or basal ganglia? Brain Res Bull. 2005;67(4):327-334. DOI: 10.1016/j.brainresbull. 2005.07.011.

68. Bergman H, Walker D, Nikolakopoulou A, SoaresWeiser K, Adams C. Systematic review of interventions for treating or preventing antipsychotic-induced tardive dyskinesia. Health Technol Assess 2017;21(43). DOI: $10.3310 /$ hta2 1430 .

69. Tarsy D, Lungu C, Baldessarini RJ. Epidemiology of tardive dyskinesia before and during the era of modern antipsychotic drugs. Handb Clin Neurol. 2011;100:601-616. DOI: 10.1016/B978-0-444-52014-2. 00043-4.

70. Kidd V. Risperidone-Induced Tardive Dyskinesia in an Autistic Child. Prim Care Companion CNS Disord. 2018;20(6).18102283. DOI: 10.4088/PCC.18102283.

71. Gorrindo P, Williams K, Lee E, Walker L, Mcgrew S, Levitt P. Gastrointestinal dysfunction in autism: parental report, clinical evaluation, and associated factors. Autism Research. 2012;5(2):101-108. DOI: 10.1002/aur.237.

72. Ming X, Brimacombe M, Wagner GC. Prevalence of motor impairment in autism spectrum disorders. Brain Dev. 2007;29(9):565-70. 